\title{
Torque Calculation With Conforming and Nonconforming Movement Interface
}

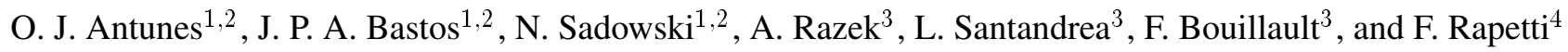 \\ ${ }^{1}$ Centro Federal de Educação Tecnologica de Santa Catarina, Florianopolis CEP 88040-900, Brazil \\ ${ }^{2}$ Universidade Federal de Santa Catarina, Departamento de Engenharia \\ Elétrica-CTC-GRUCAD, Florianopolis CEP 88040-900, Brazil \\ ${ }^{3}$ Paris VI, Paris XI Univ. and Supelec, Plateau de Moulon, Gif-sur-Yvette Cedex 91192, France \\ ${ }^{4}$ Lab. De Mathématiques, Nice-Sophia Antipolis Univ., Nice Cedex 2 06108, France
}

\begin{abstract}
This work analyzes the use of conforming and nonconforming movement techniques for torque calculation with high-order hierarchic interpolation in electrical machines thin air gaps. For the nonconforming movement formulation, the Lagrange multipliers are used. The moving band is used for the conforming case. The behavior of torque results as a function of interpolation order and air-gap thickness is discussed. In order to compare formulations, results for two thin air-gap machines are shown.
\end{abstract}

Index Terms-Hierarchic elements, Lagrange multipliers, movement, torque.

\section{INTRODUCTION}

$\mathbf{T}$ HERE ARE several works about torque calculation while considering the movement, but the use of nonconforming meshes combined with high-order interpolation has not been investigated yet. The aim of this work is to clarify the use of highorder interpolation for torque calculation; in particular, when no constraints are imposed to the rotor displacement. Many papers indicate the use of high-order interpolation to improve the torque accuracy [7]-[10]. These works, however, consider the rotor displacement step equal to the length of an element in the air gap. In other words, there is no deformation of the elements or nonconformity between the meshes. Moreover, the torque is not studied with high-order interpolation for small displacements of the rotor in small air gaps. So this subject deserves more attention. This work demonstrates that if the air gap is thin, the high-order interpolation is not recommended and the first-order one produces better results.

The torque accuracy essentially depends on the torque calculation method, the movement method, the interpolation order, the discretization, and the rotor displacement step. We choose the Maxwell stress tensor to calculate the torque, which has been used successfully when considering the movement [1]. To perform the movement the Lagrange multipliers method (LM) is used with hierarchic interpolation on the slipping interface $\Gamma$. We compare LM method with a conforming technique: the moving band (MB), used also with hierarchic interpolation [6].

\section{LAGRANGE MULTIPLIERS}

The whole domain is decomposed in two subdomains $\Omega_{a}$ and $\Omega_{b}$ connected by a sliding interface $\Gamma$.

One way to find the LM formulation is to minimize the energy functional associated with the magnetostatic problem added to a new functional to ensure the continuity of the vector potential

Digital Object Identifier 10.1109/TMAG.2006.871428 at the interface [5]. In this way, the following variational formulation is obtained:

$$
\begin{aligned}
\int_{\Gamma} \lambda v_{a} d \Gamma+\int_{\Omega_{a}} \vec{\nabla} v_{a} \cdot \frac{1}{\mu_{a}} \vec{\nabla} A_{a} d \Omega_{a} \\
=\int_{\Omega_{a}} v_{a}\left(J_{a}+\frac{\partial}{\partial x}\left[\frac{1}{\mu_{a}} B_{o y}^{a}\right]-\frac{\partial}{\partial y}\left[\frac{1}{\mu_{a}} B_{O x}^{a}\right]\right) d \Omega_{a} \\
\quad-\int_{\Gamma} \lambda v_{b} d \Gamma+\int_{\Omega_{b}} \vec{\nabla} v_{b} \cdot \frac{1}{\mu_{b}} \vec{\nabla} A_{b} d \Omega_{b} \\
=\int_{\Omega_{b}} v_{b}\left(J_{b}+\frac{\partial}{\partial x}\left[\frac{1}{\mu_{b}} B_{o y}^{b}\right]-\frac{\partial}{\partial y}\left[\frac{1}{\mu_{b}} B_{O x}^{b}\right]\right) d \Omega_{b} \\
\int_{\Gamma}\left(A_{a}-A_{b}\right) v_{c} d \Gamma=0 .
\end{aligned}
$$

In the equations above, $v_{a}, v_{b}$, and $v_{c}$ are test functions, $J_{a}$ and $J_{b}$ the current densities, and $A_{a}$ and $A_{b}$ are the vector potentials in each domain. $B_{o y}^{a}, B_{O X}^{a}, B_{o y}^{b}$, and $B_{O X}^{b}$ are remanent induction components and $\mu_{a}$ and $\mu_{b}$ are the magnetic permeabilities. $\lambda$ is identified either as the Lagrange multiplier or as the tangential component of a magnetic field on $\Gamma$. The discretization of the equations above produces a symmetric system. This system is ill conditioned and nonpositive definite. However, it is possible to reformulate the final system in a well-conditioned and positive definite form equivalent to the mortar element method. In this way, the matrices to couple the subdomains are calculated as shown in [2] for first- and second-order hierarchic interpolation on $\Gamma$.

\section{RESUltS AND DISCUSSION}

\section{A. Thin Air-Gap Synchronous Machine}

For this machine (Fig. 1), which has an air-gap thickness of $0.3 \mathrm{~mm}$, the discretization shown in Fig. 2 is used. The rotor displacement step is $0.12^{\circ}$. To overpass one edge on $\Gamma$, ten rotor displacement steps are necessary. 


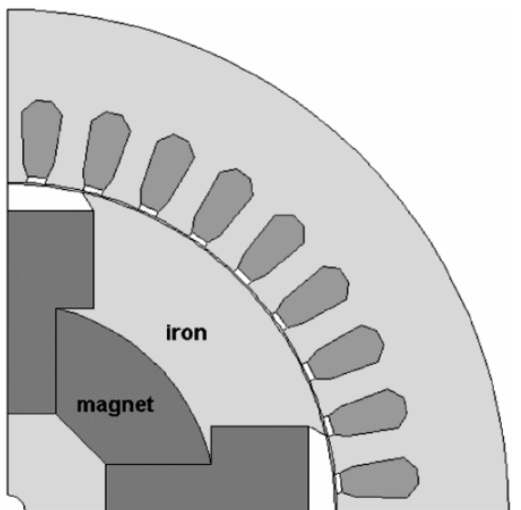

Fig. 1. Thin air-gap synchronous machine with polar pieces.

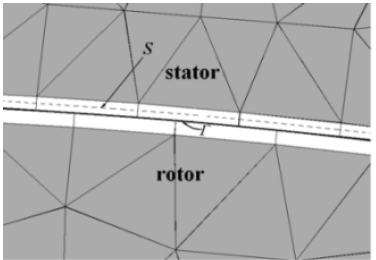

(a)

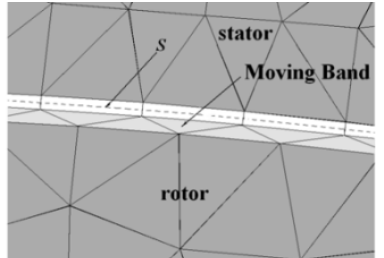

(b)
Fig. 2. Detail of the mesh for the synchronous machine with polar pieces with 81 edges on $\Gamma$, along with 1772 elements and 1015 nodes.
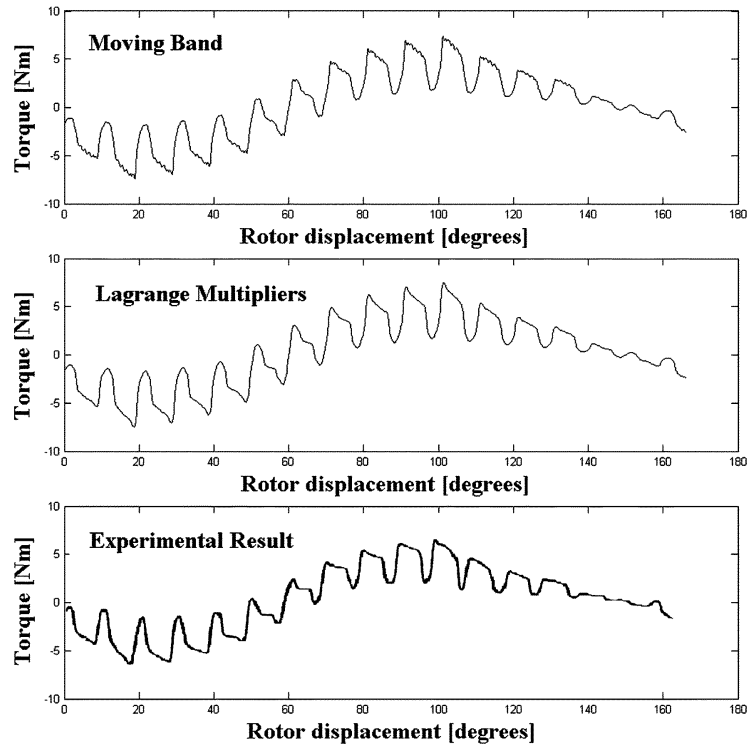

Fig. 3. Static torque for two phases connected in series with 10-A excitation. First-order interpolation on $\Gamma$ and on moving band.

The results presented in Fig. 3 for first-order interpolation, with two phases connected in series with a 10-A excitation, show good results for either LM and MB. But LM produces a more stable result as shown in the detail of Fig. 4. If the interpolation order is augmented to two (using hierarchic interpolation on $\Gamma$ and hierarchic complete elements on moving band), the results presented in Fig. 5 are obtained. In this case, against expectations, we obtain a worst result with more oscillations than with first-order interpolation.

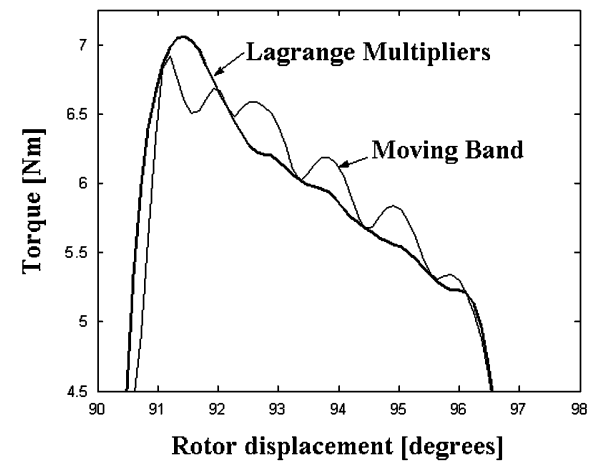

Fig. 4. Detail of Fig. 3 .
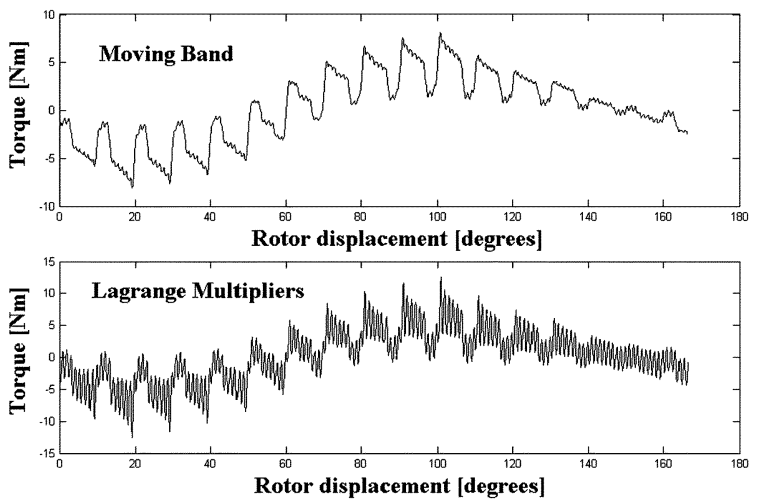

Fig. 5. Static torque for two phases connected in series with 10-A excitation. Second-order interpolation on $\Gamma$ and on moving band.

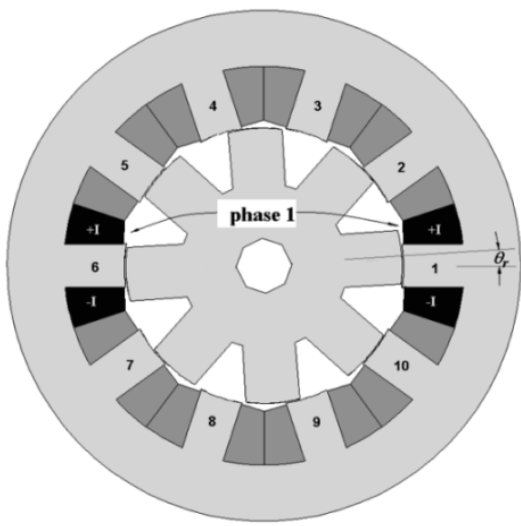

Fig. 6. Five-phase 10/8 switched reluctance motor. Phase 1 (stator poles 1 and 6) under excitation.

\section{B. Switched Reluctance Motor}

Because the torque calculation in thin air gaps may surprise, the switched reluctance motor [4] shown in Fig. 6, which has an air gap of $0.25 \mathrm{~mm}$, is also simulated.

For this machine, two discretizations (Fig. 7) are used: mesh 1 (with 2486 elements, 1605 nodes, 120 edges on $\Gamma$ and four layers of elements in the air gap) and mesh 2 (with 4861 elements, 2730 nodes, 240 edges on $\Gamma$, and 2 layers of elements in the air gap). The air-gap thickness $g$ is $0.25 \mathrm{~mm}$. The interface $\Gamma$ is placed in the middle of the air gap. The MB is placed in the layer next to the rotor side of $\Gamma$. The torque is always calculated in the layer of quadrilateral elements placed next to the stator side of $\Gamma$. The Maxwell stress tensor contour passes by 


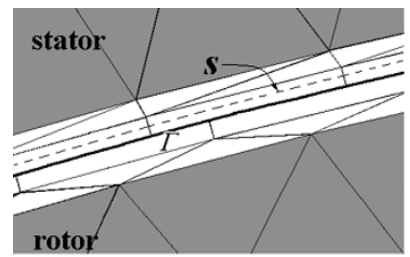

(a)

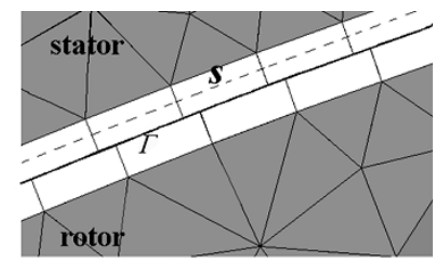

(b)
Fig. 7. Detail of (a) mesh 1 and (b) mesh 2. Slipping interface $\Gamma$ for $L M$ and Maxwell stress tensor integration surface $S$.

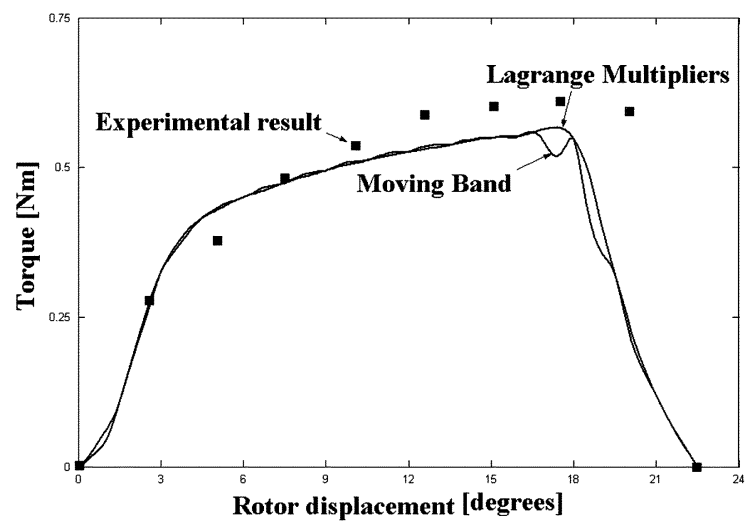

Fig. 8. Static torque for a 2-A excitation. First-order interpolation. Mesh 1.

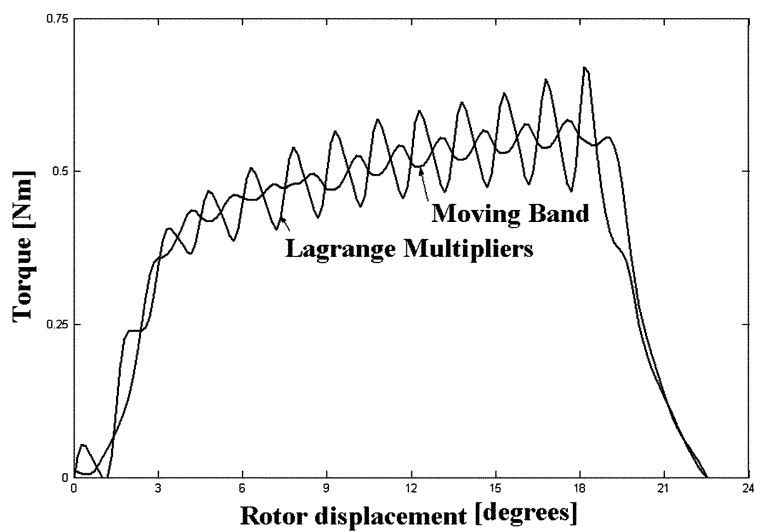

Fig. 9. Static torque for a 2-A excitation. Second-order interpolation on $\Gamma$ and on moving band. Mesh 1.

the midpoints of the quadrilateral element edges (Fig. 7). The rotor displacement step is $0.15^{\circ}$ for all results. To overpass one edge on $\Gamma 10$ (for mesh 1) and 5 (for mesh 2), rotor displacement steps are necessary.

If we compare conforming and nonconforming formulations for mesh 1 with first-order interpolation, Fig. 8 shows that the LM gives better results than the MB. However, Fig. 9 shows strong oscillations for the torque calculation with second-order interpolation, with either $\mathrm{LM}$ or $\mathrm{MB}$, as observed for the first example.

The results presented in Fig. 10 for mesh 2 show that for this more dense discretization (which has better-shaped elements, that is, minor length/width ratio) LM and MB produces equivalent results for first-order interpolation. For second-order interpolation (Fig. 11), the oscillations are reduced when compared with the result for mesh 1 (Fig. 9). But the result for first-order

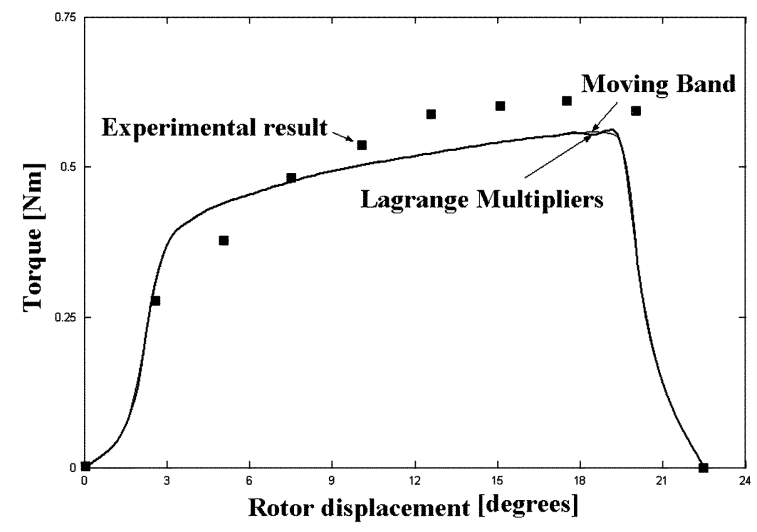

Fig. 10. Static torque for a 2-A excitation. First-order interpolation. Mesh 2.

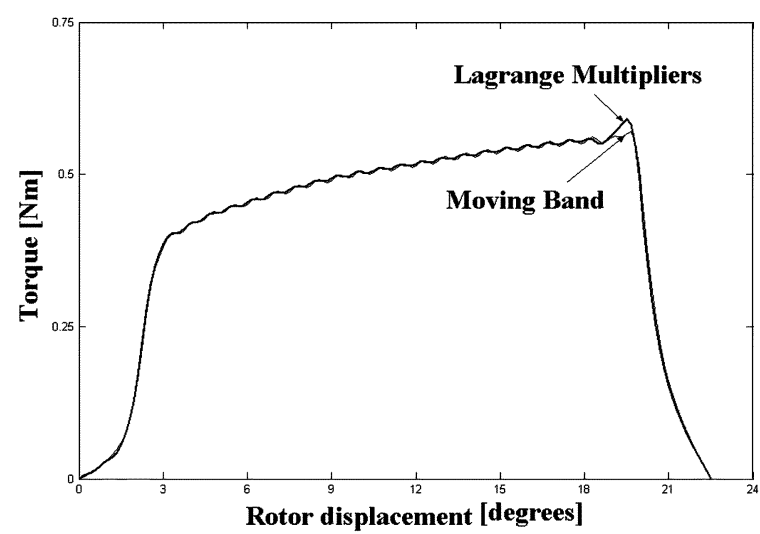

Fig. 11. Static torque for a 2-A excitation. Second-order interpolation on $\Gamma$ and on moving band. Mesh 2 .

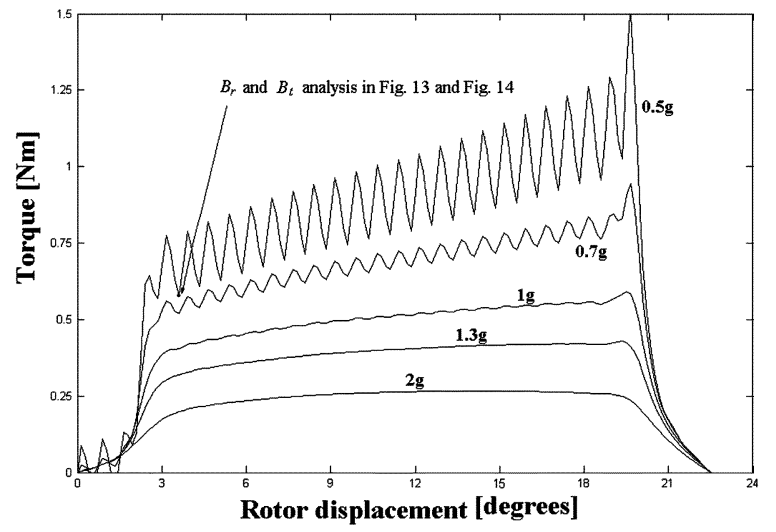

Fig. 12. Torque for various air-gap thickness. LM with second-order interpolation on $\Gamma$. Mesh 2.

interpolation (Fig. 10) is again better than with the second-order (Fig. 11).

To study the oscillations with second-order interpolation, we use the mesh 2 with LM. If we change the original air-gap thickness $\mathrm{g}$ from $0.5 \mathrm{~g}$ to $2 \mathrm{~g}$, as shown in Fig. 12, one observes that a thin air gap produces a more intense oscillation. We can accept it when analyzing the behavior of the torque results as a function of the radial $B_{r}$ and tangential $B_{t}$ components of the magnetic induction. The torque may be expressed by

$$
T=\frac{L}{\mu_{o}} \int_{S} r B_{r} B_{t} d S
$$



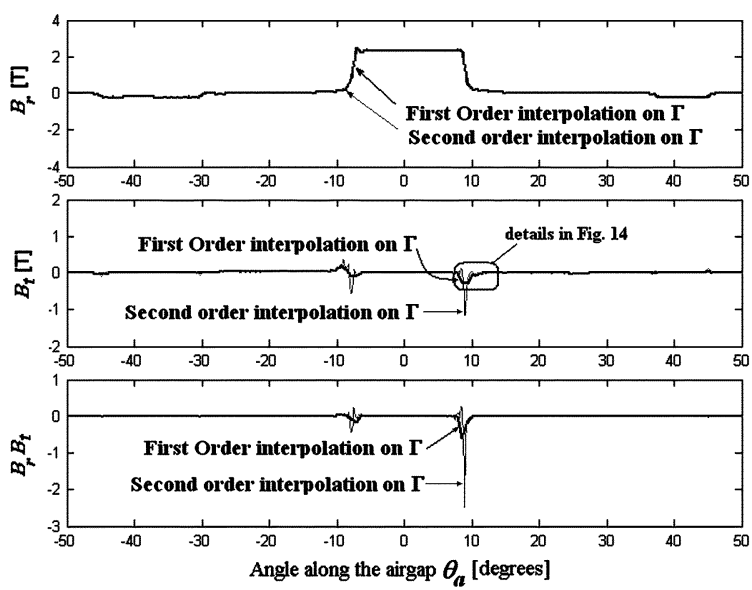

Fig. 13. $B_{r}$ and $B_{t}$ along the air gap for LM. Air-gap thickness of $0.125 \mathrm{~mm}$ or $0.5 \mathrm{~g}$. Rotor at $\theta_{r}=3.6^{\circ}$.

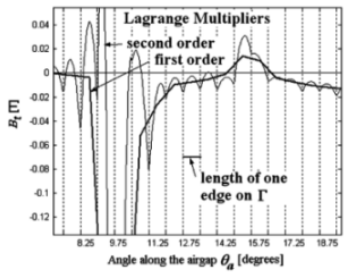

(a)

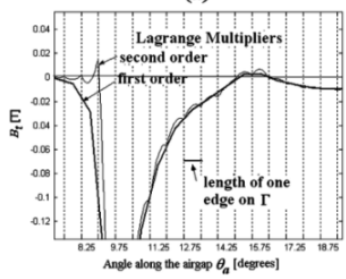

(b)

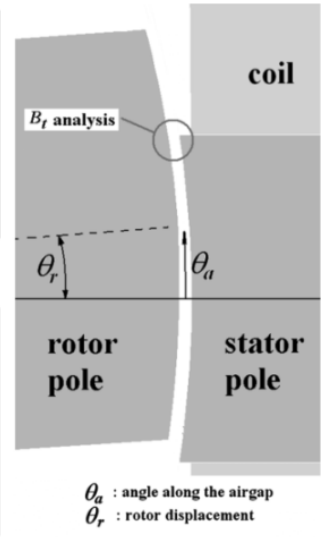

(c)
Fig. 14. $B_{t}$ along the air gap for LM. Air-gap thickness of (a) $0.125 \mathrm{~mm}$ or $0.5 \mathrm{~g}$ and (b) $0.50 \mathrm{~mm}$ or $2 \mathrm{~g}$. (c) Rotor at $\theta_{r}=3.6^{\circ}$.

where $r$ is the radius of the Maxwell stress tensor integration surface $S$, and $L$ is the axial length of the machine.

It is well known that $B_{r}$ has a regular form along the air gap. It is large at the intersection of the rotor and stator pole arcs and vanishes elsewhere (Fig. 13). But the tangential induction component $B_{t}$ has a very irregular shape. Near the pole corners, as a result of the small air gap, it has a strong variation. As shown in Fig. 14(a), when the air gap is very thin $(0.5 \mathrm{~g})$, the second-order interpolation produces strong oscillations at $B_{t}$ near the stator pole corner. This oscillation occurs either with conformity or nonconformity between the stator and rotor meshes. We can state that first-order elements, by their own nature, tend to smooth (or average) the field values, which are physically coherent. When the air gap is $2 \mathrm{~g}$, the results for firstand second-order interpolation are close [Fig. 14(b)]. In fact, we have a singular point for the solution on the stator and rotor pole corners under excitation. This singularity is higher with a thin air gap. If we consider adaptivity mesh concepts [3], the high-order elements do not handle such singularities. It is necessary for first-order elements to do so.
Its important to observe that as shown in [2], the high-order interpolation ensures more efficiently the continuity of the vector potential on $\Gamma$. This means that $B_{r}$, which is calculated with the derivative of the vector potential with respect to tangential direction, is well computed with high-order interpolation (Fig. 13 shows that $B_{r}$ for second- and first-order interpolation are very close). But, as the torque calculation depends on the product $B_{r} B_{t}$, the strong variation of $B_{t}$ near corner poles is reproduced on the torque results.

We point out that the above results were obtained for the vector potential formulation. It is likely that the same oscillations may occur with different formulations, since it seems that the oscillations are due to the high interpolation order rather than the formulations themselves.

\section{CONCLUSION}

This work shows that nonconforming LM formulation produces better torque results than $\mathrm{MB}$ with first-order interpolation when no constraints are imposed to the rotor displacement step. Moreover, against expectations, it demonstrates that second-order interpolation does not provide good accuracy for torque calculation with thin air gaps and small displacements of the rotor, which are necessary for dynamic cases or coupling with the circuit. However, as shown in [2], high-order interpolation may produce good results for electromotive-force calculation even in thin air gaps.

\section{REFERENCES}

[1] N. Sadowski, Y. Lefèvre, M. Lajoe-Mazenc, and J. Cros, "Finite element torque calculation in electrical machines while considering the movement," IEEE Trans. Magn., vol. 28, no. 2, pp. 1410-1413, Mar. 1992.

[2] O. J. Antunes, J. P. A. Bastos, N. Sadowski, A. Razek, L. Santandrea, F. Bouillault, and F. Rapetti, "Using high-order interpolation with mortar element method for electrical machines analysis," IEEE Trans. Magn., vol. 41, no. 5, pp. 1472-1475, May 2005.

[3] M. G. Vanti, "Contribuição à Adaptatividade de Malhas de Elementos Finitos Bi e Tridimensionais Com a Versão h-p," Ph.D. dissertation, Univ. Fed. Santa Catarina, Florianópolis, Brazil, 1996.

[4] T. C. Green, M. M. Khater, B. W. Willians, M. M. El-Shanawany, and A. A. Hassanein, "A five-phase switched reluctance motor: Design and performance," in IEEE Colloq. Stepper Motors Their Control, Jan. 1994, pp. $7 / 1-7 / 4$.

[5] D. Rodger, H. C. Lai, and P. J. Leonard, "Coupled elements for problems involving movement," IEEE Trans. Magn., vol. 26, no. 2, pp. 548-55, Mar. 1990.

[6] O. J. Antunes, J. P. A. Bastos, and N. Sadowski, "Using high-order finite elements in problems with movement," IEEE Trans. Magn., vol. 40, no. 2, pp. 529-532, Mar. 2004.

[7] A. N. Wignall, A. J. Gilbert, and S. J. Yang, "Calculation of forces on magnetised ferrous cores using the maxwell stress tensor," IEEE Trans. Magn., vol. 24, no. 1, pp. 459-462, Jan. 1988.

[8] T. Tärnhuvud and K. Reichert, "Accuracy problems of force and torque calculation in FE - systems," IEEE Trans. Magn., vol. 24, no. 1, pp. 443-446, Jan. 1988.

[9] W. Cai, P. Pillay, and K. Reichert, "Accurate computation of electromagnetic forces in switched reluctance motors," in Proc. 5th Conf. Electrical Machines ICEM, vol. 2, Aug. 2001, pp. 1065-1071.

[10] Z. W. Shi and C. B. Rajanathan, "A new method to improve the accuracy of maxwell stress based force calculation in computational eletromagnetic fields," in Proc. Int. Conf. Computation in Electromagnetics CEM, Apr. 1996, pp. 241-246.

Manuscript received June 28, 2005 (e-mail: orlando@grucad.ufsc.br). 